\title{
Supernova Shock Breakout from a Red Supergiant
}

\author{
Kevin Schawinski, ${ }^{1 *}$ Stephen Justham,,${ }^{1 *}$ Christian Wolf,${ }^{1 *}$ \\ Philipp Podsiadlowski, ${ }^{1}$ Mark Sullivan, ${ }^{1}$ Katrien C. Steenbrugge, ${ }^{2}$ \\ Tony Bell, ${ }^{1}$ Hermann-Josef Röser, ${ }^{3}$ Emma S. Walker, ${ }^{1}$ Pierre Astier, ${ }^{4}$ \\ Dave Balam, ${ }^{5}$ Christophe Balland, ${ }^{4}$ Ray Carlberg, ${ }^{6}$ Alex Conley, ${ }^{6}$ \\ Dominque Fouchez, ${ }^{7}$ Julien Guy, ${ }^{4}$ Delphine Hardin, ${ }^{4}$ Isobel Hook, ${ }^{1}$ \\ D. Andrew Howell, ${ }^{6}$ Reynald Pain, ${ }^{4}$ Kathy Perrett, ${ }^{6}$ Chris Pritchet, ${ }^{5}$ \\ Nicolas Regnault ${ }^{4}$ and Sukyoung K. $\mathrm{Yi}^{8}$ \\ ${ }^{1}$ Department of Physics, University of Oxford, Oxford OX1 3RH, UK. \\ ${ }^{2}$ St John's College Research Centre, University of Oxford, OX1 3JP, UK. \\ ${ }^{3}$ Max-Planck-Institut für Astronomie, Königstuhl 17, 69117 Heidelberg, Germany. \\ ${ }^{4}$ LPHNE, CNRS-IN2P3 and Universités Paris VI \& VII, 4 Place Jussieu, \\ 75252 Paris Cedex 05, France. \\ ${ }^{5}$ Department of Physics and Astronomy, University of Victoria, \\ PO Box 3055 STN CSC, Victoria, BC V8T 3P6, Canada. \\ ${ }^{6}$ Department of Physics and Astronomy, University of Toronto, \\ 50 St. George Street, Toronto, ON M5S 3H4, Canada. \\ ${ }^{7}$ CPPM, CNRS-IN2P3 and Universit Aix-Marseille II, Case 907, \\ 13288 Marseille Cedex 9, France. \\ ${ }^{8}$ Department of Astronomy, Yonsei University, Seoul 120-749, Korea.
}

*To whom correspondence should be addressed;

E-mail: kevins@astro.ox.ac.uk, sjustham@astro.ox.ac.uk,cwolf@astro.ox.ac.uk

Massive stars undergo a violent death when the supply of nuclear fuel in their cores is exhausted, resulting in a catastrophic "core-collapse" supernova. Such events are usually only detected at least a few days after the star has exploded. 


\section{Observations of the supernova SNLS-04D2dc with the Galaxy Evolution Ex- plorer space telescope reveal a radiative precursor from the supernova shock before the shock reached the surface of the star and show the initial expan- sion of the star at the beginning of the explosion. Theoretical models of the ultraviolet light curve confirm that the progenitor was a red supergiant, as ex- pected for this type of supernova. These observations provide a way to probe the physics of core-collapse supernovae and the internal structures of their progenitor stars.}

The explosive deaths of massive stars are dramatic events that seed the Universe with heavy elements $(1,2)$, produce black holes, pulsars, and the most energetic gamma-ray bursts (GRBs; 3). Their energy input can regulate the growth of galaxies (4). Even though a large amount of theoretical effort has been expended on trying to explain how the terminal collapse of a star's core leads to a luminous supernova, we do not fully understand the process by which the collapse of the core produces an outward-moving shock that leads to the ejection of the envelope (5-7). This shock heats and accelerates the stellar envelope as it passes through it. By the time the shock dissipates at the surface of the star, several solar masses of previously static envelope material are expanding at a few percent of the speed of light. At the time of core collapse, a nearby external observer equipped with a detector of neutrinos or of gravitational waves might receive a brief warning of the future explosion, but for most of the passage of the shock through the star that observer would notice no further change. Only when the shock approaches the surface does radiation diffuse far enough ahead of the shock wave to raise the temperature of the stellar photosphere. This phase is sometimes referred to as 'shock breakout', although the associated radiation is from the 'radiative precursor' of the shock, long before the shock actually reaches the surface. This radiative precursor raises the temperature of the star to $\sim 10^{5} \mathrm{~K}$ before the surface expands dramatically $(8)$. 
Shock breakouts have been inferred for a few relatively local GRBs and x-ray flashes which may involve shocks traveling through dense winds outside compact blue stars, including the recent SN 2008D (9-14). Here we describe the brightening of a red supergiant due to the theoretically predicted radiative precursor before the supernova shock reaches the surface of the star. Such observations provide information about the density profile inside the progenitor star (15) and the physics of radiative shocks, and knowledge of the spectrum of the associated ultraviolet flash has implications for the ioniziation of the circumstellar medium $(16,17)$.

Although core-collapse supernovae are expected to be most luminous around the time of shock breakout, most of this energy emerges as extreme UV or soft X-ray radiation. Hence corecollapse supernovae are typically only discovered several days after the supernova explosion near the peak of their optical light curve; observations of early light curves are rare $(18,19)$. To circumvent this problem, we exploit two complementary data sets: an optical survey to locate supernovoae, and UV data to search for serendipitously associated shock breakouts. The first is the Supernova Legacy Survey (SNLS; 20) which studies distant supernovae using data taken every 4 days at the $3.6 \mathrm{~m}$ Canada-France-Hawaii Telescope (CFHT). The second is from the Galaxy Evolution Explorer (GALEX) UV space telescope (21, 22), which took a deep 100 hour combined exposure coincident with the early-2004 SNLS data in the Cosmological Evolution Survey I"COSMOS”) field $(23,24)$. The GALEX data were taken using sub-exposures of 15 to 30 minutes over several weeks, providing data with the time resolution necessary to resolve UV-luminous events occurring before the SNLS supernovae.

One SNLS event, designated SNLS-04D2dc and confirmed as a Type II supernova from the hydrogen lines in an optical spectrum taken at the European Southern Observatory (ESO) Very Large Telescope (VLT) [supporting online material S1 (SOM text S1) (25)], shows a dramatic brightening in the GALEX near-UV images about 2 weeks before the discovery by the SNLS, consistent with shock breakout. The host galaxy appears to be a normal star-forming spiral 
galaxy at a redshift of $z=0.1854$. The supernova spectrum, Gemini host galaxy spectrum and Hubble Space Telescope image of the host are presented in 25. The optical light curve has a plateau that identifies the explosion as a Type IIP supernova (Fig. 1), suggesting a redsupergiant progenitor $(26,27)$. Because of bad weather and technical problems with the CFHT camera, there are no optical data concurrent with the UV data; however, GALEX observed the entire radiative precursor, (Fig. 2).

The GALEX light curve probes the arrival of the supernova shock at the surface of the star. We can interpret the two peaks in this light curve (Fig. 2) in terms of distinct physical processes. The first peak in the UV light curve is due to radiation traveling ahead of the shock wave. This heats the surface of the star before it begins to explode. The nearUV light curve samples the brightening caused by this precursor over 6 hours. We can compare the duration of the observed precursor with theoretical expectations by equating the photon diffusion time-scale with the time-scale for the shock to escape from the envelope. If $v$ is the shock speed and the density of the hydrogen-dominated atmosphere is $\rho$, we find $d \approx 2.5 \times 10^{11} \mathrm{~m}\left(10^{-8} \mathrm{~kg} \mathrm{~m}^{-3} / \rho\right)\left(10^{7} \mathrm{~m} \mathrm{~s}^{-1} / v\right)$ for the depth of the shock $d$ (from the surface of the star) at the time when the radiative precursor becomes visible at the surface (SOM text 3 and 4). This value for $d$ leads to a prediction for the duration of the shock precursor of $d / v=2.5 \times 10^{4} \mathrm{~s}$ for the parameters above, that is, almost 7 hours, consistent with our observations of the precursor. This indicates that the progenitor was a large star, that is, a red supergiant, as expected for the progenitor of a type IIP supernova $(26,27)$, whilst previous calculations indicated that radiative precursors from blue supergiant stars would last for minutes rather than hours (8). To model the radiative precursor, we solved simplified radiation-hydrodynamics equations for an outward-moving shock inside a stellar envelope. Figure 3 shows representative models that are consistent with the data; they require radii and envelope densities appropriate for a red-supergiant star. These models also indicate that only the initial $\sim 4$ hours of the first 
UV peak occur before the shock reaches the surface of the star.

The peak in the total luminosity of the source occurs at the time of the first UV peak, and the total luminosity monotonically decreases after this point. The temperature behind the shock is lower than the temperature at the shock front itself, which leads to a rapid drop in the luminosity of the star after shock breakout (8). The near-UV light curve in Fig. 3 shows this dip in brightness after the shock has escaped from the star.

Although the radiative precursor does cause some expansion of the star, there is little change in the stellar radius until the shock reaches the surface. Behind the shock, the radiationdominated plasma expands at almost constant velocity and cools rapidly as a result of adiabatic expansion (1). The UV light curve is now governed by the expansion of the photospheric radius (and concomitant increase in radiating surface area), the adiabatic cooling of the surface and the shift of the spectral energy distribution towards longer wavelengths, causing the second peak in the UV light curve. In the adiabatic cooling phase, the photospheric temperature $T$ is approximately inversely proportional to the photospheric radius $R$. Because for a black body this drop in $T$ causes a more rapid decrease in the luminosity $\left(L \propto T^{4} \propto R^{-4}\right)$ than the increase due to the growing surface area ( $\left.L \propto R^{2}\right)$, the total luminosity of the supernova continues to decrease. However, in the Rayleigh-Jeans portion of the spectrum the increase in the surface area of the photosphere is more important than the decrease in emission per unit area, and the luminosity at those wavelengths increases (SOM text S4.3). The observed UV luminosity rises until the peak of the blackbody spectral energy distribution nears the UV waveband. Thereafter, the UV luminosity decreases with continued adiabatic expansion and cooling. The model curves in Fig. 2 show that this simple physical description reproduces the GALEX data with parameters as expected for a red supergiant progenitor. Initial photospheric radii of 500- to 1000 solar radii $R_{\odot}$, expansion velocities of $1-$ to $2 \times 10^{7} \mathrm{~m} \mathrm{~s}^{-1}$, and initial temperatures of $\sim 10^{5} \mathrm{~K}$ match the observed fluxes well. The biggest uncertainty arises from the adopted extinction (SOM text S1); 
any increase in the NUV extinction would increase the range of preferred initial radii. Measuring precise radii of supernova progenitor stars would be a valuable constraint of the late stages in the evolution of massive stars; this require higher time resolution and more accurate temperature determinations, for example, from observing the full spectral energy distribution from $\mathrm{x}$-ray to optical. In addition, detailed light curves of radiative precursors probe the energetics of supernova shocks and the structures of the stellar envelopes through which they travel.

\section{References and Notes}

1. D. Arnett, Supernovae and nucleosynthesis. An investigation of the history of matter, from the Big Bang to the present (Princeton series in astrophysics, Princeton, NJ: Princeton University Press, 1996).

2. K. Nomoto, N. Tominaga, H. Umeda, C. Kobayashi, K. Maeda, Nuclear Physics A 777, 424 (2006).

3. C. L. Fryer, et al., PASP 119, 1211 (2007).

4. A. Dekel, J. Silk, ApJ 303, 39 (1986).

5. R. Buras, M. Rampp, H.-T. Janka, K. Kifonidis, Physical Review Letters 90, 241101 (2003).

6. A. Mezzacappa, Ann. Rev. Nucl. Part. Sci. 55, 467 (2005).

7. A. Burrows, E. Livne, L. Dessart, C. D. Ott, J. Murphy, ApJ 640, 878 (2006).

8. L. Ensman, A. Burrows, ApJ 393, 742 (1992).

9. S. Campana, et al., Nature 442, 1008 (2006).

10. A. M. Soderberg, et al., Nature 453, 469 (2008). 
11. P. A. Mazzali, et al., Nature 442, 1018 (2006).

12. L.-X. Li, MNRAS 375, 240 (2007).

13. G. Ghisellini, G. Ghirlanda, F. Tavecchio, MNRAS 382, L77 (2007).

14. L.-X. Li, htttp://arxiv.org/abs/0803.0079 (2008).

15. A. J. Calzavara, C. D. Matzner, MNRAS 351, 694 (2004).

16. C. Fransson, P. Lundqvist, ApJ 341, L59 (1989)

17. P. Lundqvist, ApJ 511, 389 (1999)

18. M. Stritzinger, et al., AJ 124, 2100 (2002).

19. R. M. Quimby, et al., ApJ 666, 1093 (2007).

20. P. Astier, et al., $A \& A 447,31$ (2006).

21. D. C. Martin, et al., ApJ 619, L1 (2005).

22. P. Morrissey, et al., ApJS 173, 682 (2007).

23. N. Scoville, et al., ApJS 172, 1 (2007).

24. M. A. Zamojski, et al., ApJS 172, 468 (2007).

25. More details are available in the supporting material on Science Online.

26. S. W. Falk, W. D. Arnett, ApJ 180, L65 (1973).

27. S. J. Smartt, et al., Science 303, 499 (2004). 
28. KS is supported by the Henry Skynner Junior Research Fellowship at Balliol College, Oxford. SJ acknowledges support by STFC \& Global Jet Watch, CW and ESW by STFC and MS by the Royal Society. This work is supported by Acceleration and Basic research programs of MOST/KOSEF to SKY. We gratefully acknowledge use of data from the NASA GALEX (Galaxy Evolution Explorer) satellite, the Canada-France-Hawaii Telescope (CFHT), the ESO Very Large Telescopes (VLT), the Gemini Observatory and the Hubble Space Telescope. 


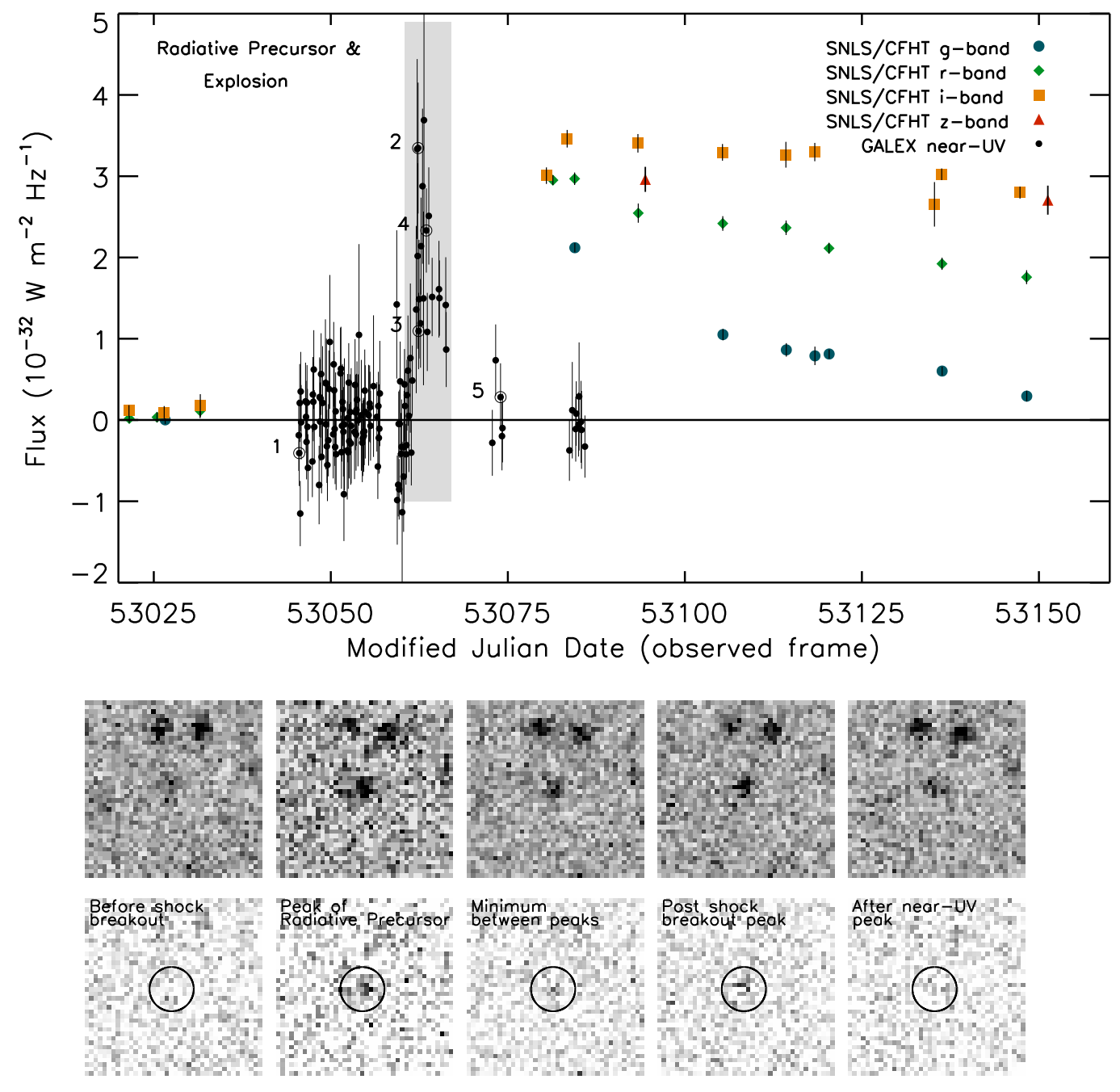

Fig. 1. Composite of the optical SNLS and the UV GALEX light curves, or observed fluxes as a function of modified Julian date. All fluxes are host galaxy subtracted and are not corrected for internal extinction. The gray box indicates the time of the radiative precursor. The points highlighted by circles indicate five phases of the radiative precursor in the UV, as observed by GALEX. These five phases are illustrated by a time sequence of original near-UV images (upper row, $1 \times 1$ arcmin) and difference images (lower row, with a pre-SN image subtracted) to emphasize the transient source. Note the drop from point 2 to the minimum at 3 and the rise to 4, clearly visible in the GALEX images. The lack of optical data during the UV event is due 
to both poor weather conditions and technical problems. Both GALEX and SNLS light curves are available as Tables in the SOM. 

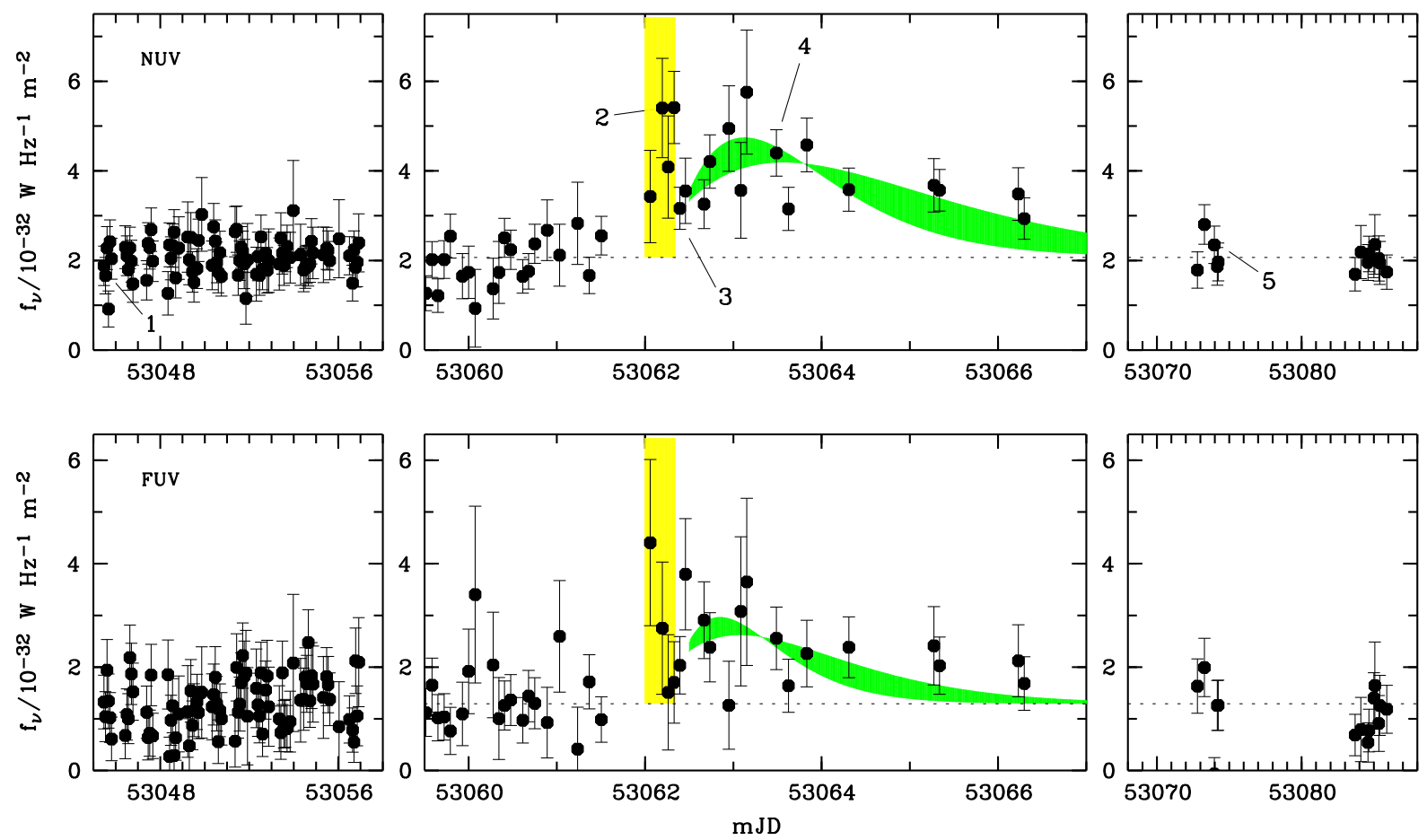

Fig. 2. The GALEX near-UV and far-UV flux against time (modified Julian date in days). This is a zoomed-in version of the shaded time range of Fig. 1 and we mark the same five data points. The background levels are shown before and after the supernova (left and right panels), and the central panels show the event itself. The radiative precursor is highlighted in yellow. Models for the post-explosion expansion are shaded in green; these models assume an initial photospheric radius of 500-1000 $R_{\odot}$. The width of the green band is due to the range of assumed expansion velocities $\left(1-2 \times 10^{7} \mathrm{~m} \mathrm{~s}^{-1}\right)$. These models assume adiabatic free expansion of a radiationdominated plasma and black-body emission from a well-defined photosphere (see text). The models were only fitted to the NUV data, but are also consistent with the FUV data. 

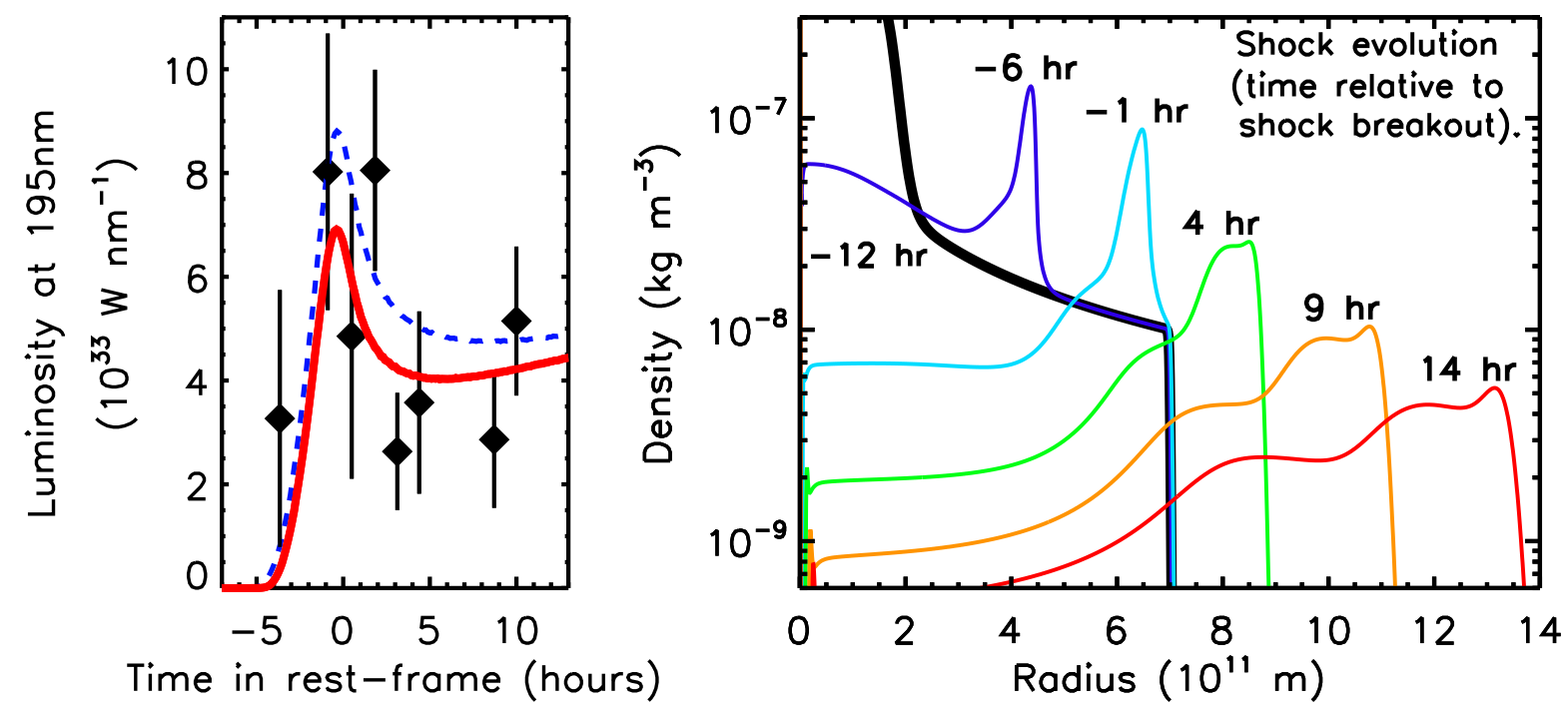

Fig. 3. Left: representative model precursor light curves compared to the precursor data (diamonds with error bars). The solid red curve represents the light curve produced from a model with an initial radius of $7 \times 10^{11} \mathrm{~m}\left(\approx 1000 R_{\odot}\right)$ and an initial density distribution $\propto 1 / r$. The dashed curve in the left hand panel shows an identical model except that the initial radius was $10^{12} \mathrm{~m}$; the absolute normalisation of these luminosities is uncertain to factors of order unity (see supplementary material for details). The zero-point of the time axis is approximately at shock breakout. Right: the time evolution of the internal density profile for the model producing the solid light curve in the left panel. Around 4 hours before the shock has reached the surface the UV luminosity has already begun to rise. 


\section{Supplementary Online Material}

\section{S1 Spectra of Supernova and Host Galaxy}

SNLS searches for high-redshift supernovae (SNe) with the goal of measuring about 500 distant Type Ia supernovae (SNe Ia) for use in studying dark energy. The volume and depth of these observations also allows detection of a large number of core-collapse SNe. While the dedicated spectroscopic follow-up of SNLS is prioritised for the SN Ia candidates, a spectrum for SNLS04D2dc (located at RA = 10:00:16.7, Dec $=+02: 12: 18.52$, J2000) was obtained on 2004-03-19 (a few days after discovery) at the European Southern Observatory Very Large Telescope 1 The spectrum provided an excellent match to a type II SN (Fig. S1) located in a star-forming galaxy at a redshift $z=0.1854$. A second spectrum obtained in early 2008 at the Gillett Gemini North Telescope after the SN had faded confirms the presence of strong emission lines in the spiral host galaxy. In Figs. S1 and S2, respectively, we present the spectra and the Hubble Space Telescope F814W image of the host galaxy from the COSMOS survey.

We have extracted a spectrum at the location of the SN inside the galaxy to estimate the host galaxy extincton based on the Balmer decrement of the galaxy spectrum. We used the SMC reddening law $(S 1)$ to estimate the extinction in the GALEX bands, which is appropriate for most star-forming galaxies at most redshifts including lower-mass galaxies at low redshift. The colour excess $E_{B-V}$ was determined from the Balmer decrement at the location of the $\mathrm{SN}$ and gives $E_{B-V}=0.14$, with implied extinction on the GALEX UV filters of $A_{\mathrm{NUV}}=1.45 \mathrm{mag}$ and $A_{\mathrm{FUV}}=2.39 \mathrm{mag}$. The largest source of uncertainty in the extinction measurement is probably its low spatial resolution. From the variation in $E_{B-V}$ we see between stars within $\sim 1 \mathrm{kpc}$ of the Sun, we estimate that the uncertainty in the absolute extinction of the supernova

\footnotetext{
${ }^{1}$ ESO/VLT large programme number 171.A-0486.
} 
could be as much as a factor of two.

An alternative way to estimate the extinction of this supernova is to use the empirical relation found by $(S 2)$ for a set of IIP supernovae, based on the colours during the optical plateau. Using the restframe V-I colour at day 50 of 0.54 , their relation is broadly consistent with the level of extinction for this supernova which we measure from the emission lines, though their relation is also consistent with no extinction for this supernova.

\section{S2 GALEX Data Reduction}

We determined the photometry for the NUV light curve by processing image frames of $10^{\prime} \times$ $10^{\prime}$ size centered on the SN using the MPIAPHOT package (S3). We co-added a selection of 161 frames with a reasonably Gaussian PSF to obtain best-possible position estimates when searching objects with SExtractor (S4). We then transformed the coordinates of the object list back into the coordinate frames of each single exposure and measured fluxes centered on the projected object positions.

We suppressed the propagation of variations in the PSF (presumably mostly due to focus drifts) into the photometry by making sure that we always probe the same physical footprint $f(x, y)$ of any object in all exposures irrespective of the PSF $p(x, y)$. Here, the footprint $f(x, y)$ is the convolution of the PSF $p(x, y)$ with the aperture weighting function $a(x, y)$. If all three are Gaussians, an identical physical footprint can be probed even when the PSF changes, simply by adjusting the weighting function $a(x, y)$ for each frame. We chose to measure fluxes on a footprint of 7.5 " FWHM, so that on average $p \approx a$, which optimizes the signal-to-noise ratio of point sources.

Hence, we measure the PSF on each individual frame, choose the weighting function needed to conserve the footprint and obtain the flux on the footprint. Individual frames are normalized to each other using the count rates of the 15 brightest non-variable objects. Fluxes from indi- 
vidual frames are averaged for each object and the flux error is derived from the scatter. Thus, it takes not only photon noise into account, but also sub-optimal flat-fielding, errors in the background determination and uncorrected detector artifacts. All fluxes are finally calibrated to the GALEX photometric catalogues using the brightest stars. As such the MPIAPHOT aperture fluxes correspond to total fluxes for point sources, but underestimate them for extended sources. In this way, we have measured the NUV flux from the SN alone (the excess flux over the host level) in a physically non-variable aperture, with ideal $\mathrm{S} / \mathrm{N}$, and correctly calibrated.

Fig. S3 shows the distribution of NUV object flux scatter vs. mean fluxes for $\sim 1000$ objects detected in the selected area. Only two objects, the SN and a QSO, appear as significantly variable by showing more flux scatter among the frames than expected from Poissonian noise. When sources are fainter than the background, their flux scatter is driven by constant noise in the estimated background (horizontal arm). The scatter of bright sources is dominated by Poisson fluctuations in the source flux, so these form a steep arm at slope 1/2 (in a log-log plot).

Fig. 2 shows part of the resulting light curve; a large group of frames obtained two years after the SN event is omitted as it shows just the host galaxy light at the same level and scatter as before the event. The dip between peaks is statistically significant; by summing the NUV making up the first peak, the dip and the start of the second peak, we determine that the significance of the drop is $2.77 \sigma$.

The FUV images have been processed in a similar fashion except that the low count rates are a challenge for determining the PSF, background and normalization of individual frames. The resulting FUV fluxes should be considered uncertain at a $+/-30 \%$ level in each frame. For this reason the FUV light curve is only shown to indicate that excess fluxes are observed exactly at the time of the NUV event, but the FUV data are not included in the model fit for the photospheric expansion. The model curves are plotted in the FUV panels just as they are predicted by the NUV fit. 
Given that we find two variable sources in an area of $10^{\prime} \times 10^{\prime}$ the probability for a chance coincidence of a random variable with the location of the host galaxy (known to 1" x 1") is $2: 360,000$. However, if you restricted yourself to the short time period near the supernova, this probability would shrink even further.

We use a cosmology consistent with the WMAP 3 year results and assume a Hubble Constant $H_{0}=70$.

\section{S3 Analytic Estimates for the Radiative Precursor}

Here we give more details about the derivation of the scaling relations given in the main text.

\section{S3.1 Estimated Depth and Duration}

First we estimate the depth of the shock $d$ within the star at the start of the radiative precursor. This is defined by equating the photon diffusion time-scale $\tau_{\text {diff }}$ with the time-scale for the shock to escape $\tau_{\mathrm{s}}$, where

$$
\begin{aligned}
\tau_{\text {diff }} & \approx \frac{3 d^{2}}{\alpha l c}, \\
\tau_{\mathrm{s}} & \approx \frac{d}{v}
\end{aligned}
$$

and $l$ is the photon mean free path, $v$ is the shock speed, $c$ is the speed of light and $\alpha$ is a constant which depends on the density profile of the progenitor (S5). The value of $\alpha$ is $\approx 10$ for a uniform density sphere or $\approx 30$ or $\approx 90$ if the density profile of the sphere drops as $r^{-1}$ or $r^{-2}$, respectively (S5). If the system is better modeled by a thin shell than a uniform sphere, then $\alpha=1$. We adopt $\alpha \approx 10$ as we assume that the depth of the shock at the time of the precursor is not negligible, and that the density profile in the relevant part of the envelope is roughly constant. This seems to be consistent with careful models of red-supergiant envelopes (S6). 
Equating $\tau_{\text {diff }}$ with $\tau_{\mathrm{s}}$ and rearranging produces

$$
d \approx \frac{\alpha c l}{3 v}
$$

in which we will substitute the mean free path for an opacity $\kappa$ dominated by electron scattering in a hydrogen atmosphere $\kappa_{\mathrm{es}, \mathrm{H}}$ of density $\rho$. This gives

$$
d \approx 2.5 \times 10^{11} \mathrm{~m}\left(\frac{\alpha}{10}\right)\left(\frac{\kappa}{\kappa_{\mathrm{es}, \mathrm{H}}}\right)^{-1}\left(\frac{\rho}{10^{-8} \mathrm{~kg} \mathrm{~m}^{-3}}\right)^{-1}\left(\frac{v}{10^{7} \mathrm{~m} \mathrm{~s}^{-1}}\right)^{-1}
$$

for the depth of the shock at the time when the radiative precursor becomes visible at the surface. In the main text, we have omitted the dependence on $\alpha$ and $\kappa$ for simplicity. Note that the depth estimated here is a very good match to the depth of the shock in Fig 3. at the start of the radiative precursor.

This value for $d$ is $\sim 350 R_{\odot}$. As the progenitor's radius must be larger than $d$, we require a red supergiant, as expected for a IIP SN. The duration of the radiative precursor should be $d / v=2.5 \times 10^{4} \mathrm{~s}$ for the parameters above, i.e. almost 7 hours and thus in good agreement with our observations. To be precise, we should increase this duration by the light travel time across the disc, but this constitutes a fairly small correction. As stated in the main text, the duration of shock breakout from a blue supergiant is completely incompatible with our observations (see also $S 7)$.

Comparison with our numerical simulations and observations suggests that a lower value of $\alpha$ might provide a more precise match to the data, as the precursor itself lasts only $\approx 4$ hours before shock breakout. A value of $\alpha \approx 5$ is a reasonable value, intermediate between $\alpha=1$ for a thin shell and $\alpha=10$ for a sphere of uniform density.

The density adopted here is consistent with the envelopes of red-supergiant models used in previous SN modeling, although they are towards the lower end of expectations $(S 6, S 8)$. Note that the modelling of red supergiant envelopes is uncertain. Convection becomes inefficient near the surface of such stars, rendering mixing-length theory inadequate. In addition, the boundary 
conditions of red giant models should be carefully matched to models of the star's wind in order to faithfully model the density profile of the envelope (S6).

\section{S3.2 Estimated Energy Release}

We can estimate the total energy released in the radiative precursor $E_{r p}$ in a way almost independent of the density profile. The total energy radiated during the passage of a radiation-dominated shock with velocity $v$ through a mass $M$ is $\approx(18 / 49) M v^{2}$ and we can approximate the mass as $M \approx 4 \pi R^{2} \rho d$, where $\rho$ and $d$ are again the density of the outer envelope and the depth of the shock when the radiative precursor is first visible. We take $R$ to be the radius of the star, which is a good approximation if $R \gg d$. We can now replace $d$ using Eq. (S2) and then use $\rho l=\kappa^{-1}$ to obtain

$$
\begin{gathered}
E_{\mathrm{rp}} \approx\left(\frac{18}{49}\right) 4 \pi R^{2} \frac{\alpha c v}{3 \kappa} \\
E_{\mathrm{rp}} \approx 2.3 \times 10^{42} \mathrm{~J}\left(\frac{R}{10^{12} \mathrm{~m}}\right)^{2}\left(\frac{\alpha}{10}\right)\left(\frac{v}{10^{7} \mathrm{~m} \mathrm{~s}^{-1}}\right)^{-1}\left(\frac{\kappa}{\kappa_{\mathrm{es}, \mathrm{H}}}\right)^{-1} .
\end{gathered}
$$

Dividing this by a duration of $\sim 10^{4}$ s predicts a mean luminosity of $\sim 10^{38} \mathrm{~W}$, consistent with our extrapolation from the observed UV flux using a temperature of $\sim 10^{5} \mathrm{~K}$ (see section S4.2).

\section{S4 Numerical Light Curve Models}

We have produced UV light curves for both the radiative precursor and the post-shock-breakout adiabatic expansion. Our simple numerical models use the physics essential to the respective phases. They naturally produce light curves consistent with the data using the expected physical input and the very minimum of parameter fitting.

The following models both produce a spectral energy distribution (SED). The effects of cosmological redshift were applied to the SED and the time axis of the expansion. The full GALEX filter functions were used to define the NUV and FUV bands. The extinction of the UV 
emission probably constitutes the biggest uncertainty in our models. We have used measured values for the extinction (see section $\mathrm{S} 1$ ) such that the NUV flux which reaches us is $\approx 1 / 3.8$ of the emitted flux, and the FUV flux is $\approx 1 / 9.0$ of the unextinguished value. However, even the 'local' measurements of the host galaxy's extinction are not guaranteed to be exactly the same as those which would be appropriate for the SN (see section $\mathrm{S} 1$ ).

\section{S4.1 The Radiative Precursor}

To model the radiative precursor we have written a bespoke one-dimensional, two-temperature, hydrodynamic code. The code is Eulerian; we have used 800 radial cells across the initial model (hence with a typical cell size of $\sim 10^{9} \mathrm{~m}$ ) and 4000 cells in total. Radiation transport is handled during each timestep by solving the diffusion equation for the internal energy $U_{\text {rad }}$ of radiation within the moving radiation-dominated plasma, where the the diffusion constant is $c /(3 \rho \kappa)$ and we assume that the opacity $\kappa$ is mostly due to electron scattering inside a hydrogen-dominated plasma. In addition to elastic Compton scattering, the radiation treatment includes Compton cooling and bremsstrahlung (S9), and the hydrodynamics naturally incorporates advection and adiabatic cooling. When a temperature is required for the emission model we take the fourth root of the energy density of the radiation, $T=\left(c U_{\text {rad }} / 4 \sigma\right)^{(1 / 4)}$.

The initial conditions specify a density distribution for the cold $(10 \mathrm{eV})$ envelope and for the hot $(1 \mathrm{MeV})$ core. The core properties were chosen such as to eventually produce a shock moving with a characteristic velocity of $1-2 \times 10^{7} \mathrm{~m} \mathrm{~s}^{-1}$. Note that this means that the first model in Fig. 3 is not at core collapse, or directly taken from a stellar evolution code. The times in Fig. 3 are approximately relative to shock breakout.

This model includes the essential physics to describe the motion of the shock through the star. However, modelling the exact spectral energy distribution and luminosity of the radiative precursor would be a much more complex task, partly as during shock breakout the luminosity 
may be augmented by some non-thermal emission (S10). To the accuracy currently demanded by the data in the UV waveband, it seems reasonable to approximate the emission as black-body.

We do not attempt a full solution of the radiative-transfer problem, but note that the photons which have diffused to the surface will carry a temperature which was imprinted on them deeper in the star, as the opacity is largely due to elastic scattering from electrons. We estimate that the typical photon will have diffused from an optical depth of $c / 3 v_{\text {shock }}$ and therefore adopt this depth to define the characteristic temperature for the black body. However, the radiation flux at the surface will be somewhat lower than at the optical depth where the photons originated (as the diffusion speed is rather lower than the speed of light). Our models indicate that the intensity of emission will be lower by up to a factor of five than would be expected for this black-body temperature over the entire surface. Given those uncertainties, the light curves in Fig. 3 have been roughly rescaled down to show that expected shapes are consistent with the data. The solid light curve in Fig. 3 has been multiplied by $1 / 2.5$ and the dashed by $1 / 4$, both within the uncertainties of this modelling.

In addition to any simplifications introduced in our modelling, we note that most of the luminosity during the radiative precursor will be emitted at higher energies than we directly observe; it is unclear whether a significant fraction of those shorter-wavelength photons will lead to the production of UV radiation through some indirect route.

This model has the significant benefit of physical clarity, but more detailed and complex work will be needed to fully exploit future observations.

\section{S4.2 After Shock-Breakout}

After the radiative precursor, GALEX has observed the early stages of the SN's expansion. As summarized in the main text, this phase is relatively simple to understand. The radiationdominated plasma expands freely (with almost constant velocity) and cools adiabatically, hence 
$T \propto 1 / R(S 5)$. The energy source is the internal energy of the plasma; radioactivity is only relevant much later. Our model also assumes that the emission can be approximated by a singletemperature black body, and this rest-frame SED is converted into an observer-frame GALEX UV flux as described at the start of section S4. The assumption that there is a well-defined photosphere is reasonable for the very early stages of expansion which we have observed.

The model light curves represented in Fig. 2 were produced by this physical model of free adiabatic expansion. The initial radius and expansion velocity were set by hand to a range of expected values for the progenitor of such a type IIP SNe. Then the initial temperature and time of explosion were fitted such that the $\chi^{2}$ parameter with respect to the NUV data was minimized. The FUV data was not fitted, but the model light curves are still consistent with the data. This second phase, visible in the UV after the radiative precursor, constrains the dimensions of the precursor independently of the precursor model.

As the envelope cools and becomes less dense, the later behavior and definition of the photosphere is more complex. In particular, the plateau in the late-time optical light curve that is characteristic of type IIP SNe is thought to be due to such complications. During the plateau, the effective photosphere moves inwards in mass but remains at an almost constant radial position and temperature. The color of the $\mathrm{SN}$ is not precisely constant during the plateau (see Fig. 1); the emission during that stage is not from a simple black body.

\section{S4.3 The Second Peak in the UV Light Curve}

Although the physics governing the phase of adiabatic expansion is simpler than in the previous or subsequent epochs, we find that the main feature of this era is sometimes not intuitively understood. Fig. S4 demonstrates how the moving peak of the black-body spectral energy distribution allows the luminosity in a particular waveband to change non-monotonically, even though the total luminosity is always decreasing. 
As an alternative way to visualise this, we can write an equation for the black body luminosity as a function of temperature and frequency, $L(T, \nu)$, neglecting numerical factors, as

$$
L(T, \nu) \propto \nu^{3} R^{2} \frac{1}{e^{h \nu / k T}-1},
$$

where $h$ and $k$ are Plank's and Boltzmann's constants, respectively. In the Rayleigh-Jeans limit this becomes

$$
L(T, \nu) \propto \nu^{2} T R^{2} \propto \nu^{2} \frac{1}{T}
$$

where we have used the fact that $R \propto 1 / T$ in the phase of adiabatic expansion (S5). So in the Rayleigh-Jeans portion of the spectrum, the luminosity at a given frequency from the surface of an adiabatically expanding optically-thick sphere is inversely proportional to the temperature. The luminosity at that wavelength begins to decline once the peak of the spectrum moves close to the UV band and the Rayleigh-Jeans approximation is no longer valid at that frequency. Fig. S4 suggests that the second peak occurs for a given $\nu$ when $h \nu \approx 2 k T$.

Note that this second peak in the light curve (the 'adiabatic peak') occurs at a different time for each frequency. The maximum bolometric luminosity occurs at the only time when the luminosity in all wavebands peaks simultaneously, and the secondary UV maximum is not coincident with a peak in the visible light output. Furthermore, once the phase of adiabatic expansion is over, our analysis is no longer valid (e.g., the optical plateau is governed by completely different physics).

There seems to be some confusion over whether the observations in (S11) have resolved shock breakout in the type Ib/c SN 1999ex. Note that the cadence of their observations is easily long enough to miss the radiative precursor that we have observed. The progenitor of 1999ex would not be a red supergiant but a much more compact star. The duration of the radiative precursor preceding shock emergence from such a compact star should be much shorter than that which we observe. We thus find it extremely unlikely that (S11) observed the shock emerging 
from within the star. However, we note that the timescale of the early dip they observe in the $U$ band is consistent with the timescale we find for the phase of adiabatic cooling.

\section{S5 Suggestions for Further Observations}

Finding more events like SNLS-04D2dc will help further our understanding of core-collapse SNe. We provide some general ideas on how a larger sample of such events might be obtained. The starting place for any such survey design must be the assumption that SNLS-04D2dc was a normal event; we assume that a UV light curve of the same absolute magnitude in the near-UV is associated with all - or at least a majority of - Type II SNe. If this is the case, we must further consider the dust extinction in the host galaxies and the locations of Type II SNe. The near-UV is much more sensitive to dust extinction than optical wavelengths, and so it is conceivable that a SN Type II occurring in a heavily extincted host galaxy is detected in optical filters, but remains undetected in the $\mathrm{UV}$.

Taking our discovery of SNLS-04D2dc as the starting point, we can estimate the rate of such events in a SN survey similar to SNLS. SNLS-04D2dc is at the edge of what is detectable in single GALEX visits, so we can expect that no similar events will be detectable beyond a redshift of $z \sim 0.2$ (the host galaxy of SNLS-04D2dc is at $z=0.1854$ ). We can compute the expected SN Type II rate from the typical cosmic star formation density out to $z \sim 0.2$ probed by one GALEX field of view (circular, $1.4 \mathrm{deg}$ diameter). The co-moving volume probed by this field of view is $\sim 8 \times 10^{4} \mathrm{Mpc}^{3}$. Combining this with a star formation rate density of $\rho_{\mathrm{SFR}} \sim$ $0.03 \mathrm{M}_{\odot} \mathrm{yr}^{-1} \mathrm{Mpc}^{-3}(S 12)$ yields a total star formation rate probed of $\sim 2.5 \times 10^{3} \mathrm{M}_{\odot} \mathrm{yr}^{-1}$. Assuming a core-collapse rate of 1 per century per $4 \mathrm{M}_{\odot} \mathrm{yr}^{-1}$, this results in 6.2 events per year per field of view. There are of course substantial uncertainties in this estimate.

Thus, mounting a substantial GALEX observational effort covering e.g 4 fields-of-view continuously would yield about 2 such events per month. A dedicated optical photometry and 
spectroscopic survey for prompt follow-up of any detected events would also be necessary. The resulting deep GALEX image will also be scientifically useful for other purposes. The key feature of any future similar observations must remain the high cadence. GALEX with its ultraviolet capability and 90-minute orbit is the most suitable platform for further research into the radiative precursors of supernovae.

\section{References and Notes}

S1. Y. C. Pei, ApJ 395130 (1992).

S2. P. Nugent, et al., ApJ 645, 841 (2006).

S3. H.-J. Röser, K. Meisenheimer, A\&A 252, 458 (1991).

S4. E. Bertin, S. Arnouts, $A \& A S$ 117, 393 (1996).

S5. D. Arnett Supernovae and nucleosynthesis. An investigation of the history of matter, from the Big Bang to the present Princeton University Press (1996)

S6. A. Heger, PhD thesis, Max-Planck-Institut für Astrophysik (1998).

S7. C. D. Matzner, C. F. McKee, ApJ 510, 379 (1999).

S8. S. W. Falk, W. D. Arnett, ApJS 33, 515 (1977).

S9. R. A. Chevalier, R. I. Klein, ApJ 234, 597, (1979).

S10. R. I. Klein, R. A. Chevalier, ApJ 223, L109 (1978).

S11. M. Stritzinger, et al., AJ 124, 2100 (2002).

S12. K. Glazebrook, et al., ApJ 587, 55 (2003). 


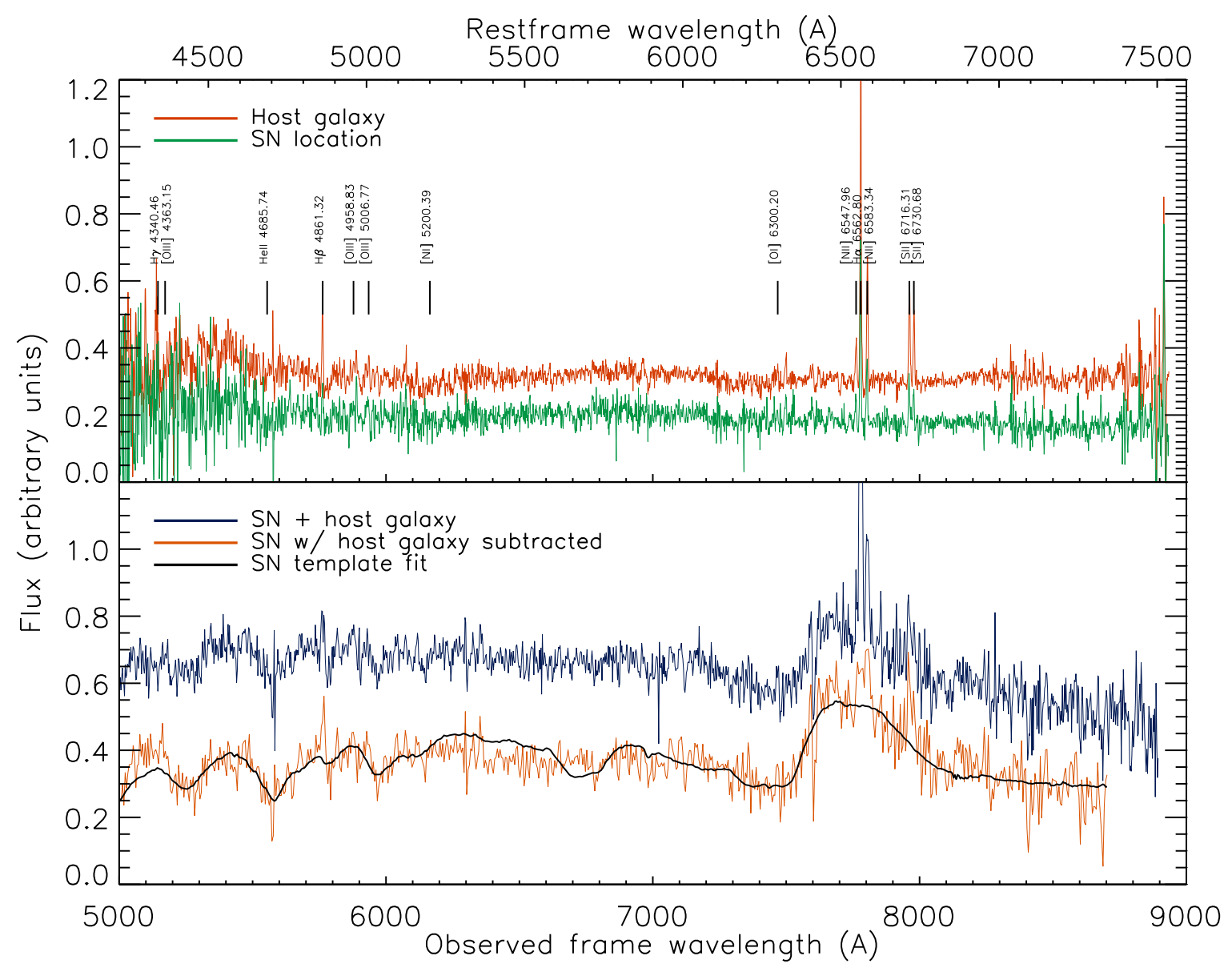

Fig. S1. In the upper panel, we show the Gemini spectra of the host galaxy as a whole and of the $\mathrm{SN}$ location. Both show strong emission lines as expected of a spiral galaxy, including $\mathrm{H} \alpha$ and $\mathrm{H} \beta$ from which we estimate the extinction affecting the $\mathrm{SN}$. In the lower panel we show the VLT spectrum of the SN, prior to and after the subtraction of a host galaxy template, together with an SN Type IIP template identifying the SN type. 


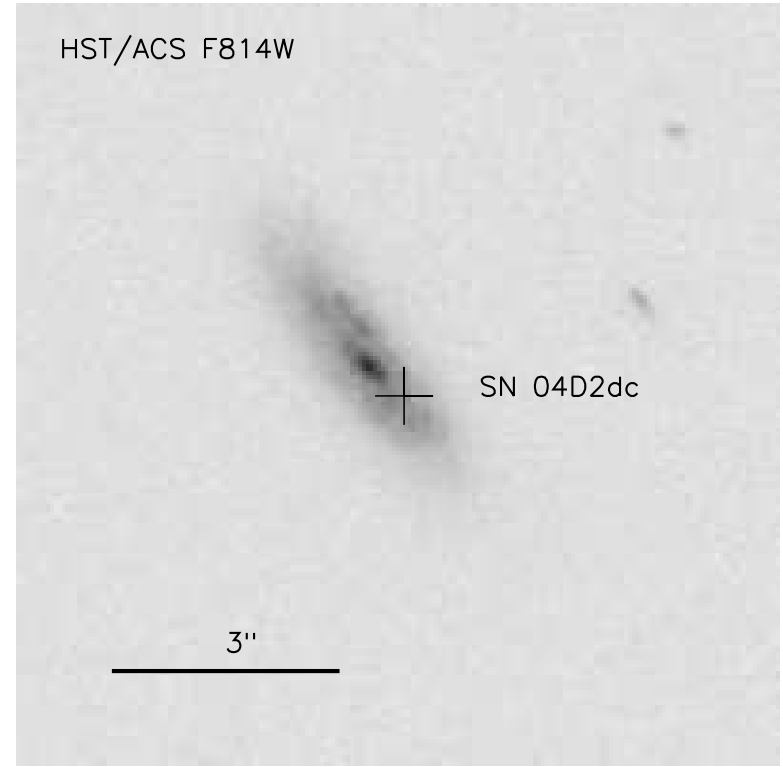

Fig. S2. The Hubble Space Telescope F814W-band image of the host galaxy from the COSMOS survey. We indicate the position of the $\mathrm{SN}$ and the image scale in arcseconds. 


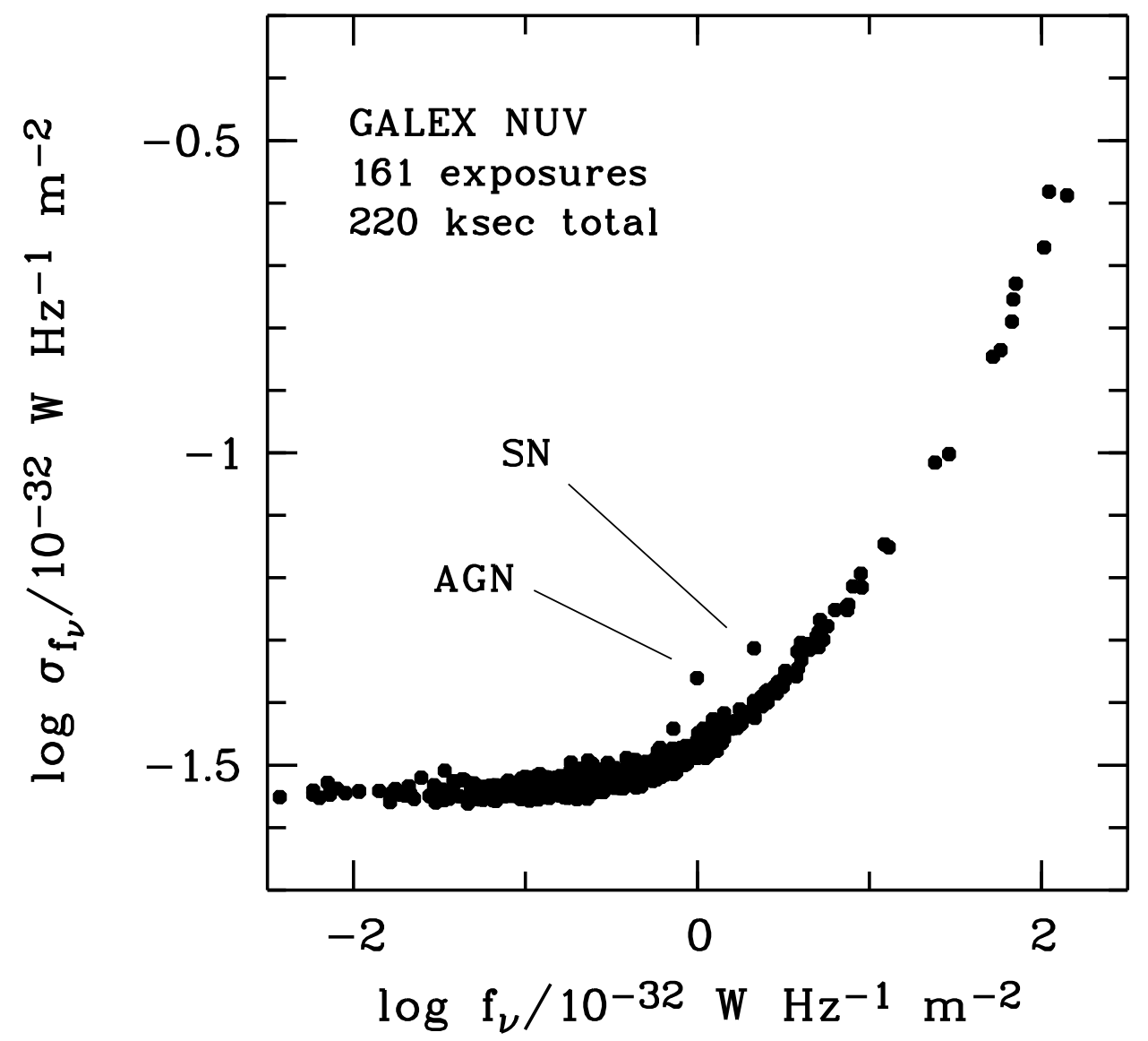

Fig. S3. Mean fluxes $f_{\nu}$ determined from 161 individual measurements and scatter $\sigma_{f_{\nu}}$ among them. Two objects out of $\sim 1000$ are clearly variable as evidenced by their increased scatter, one of which is the SN. 

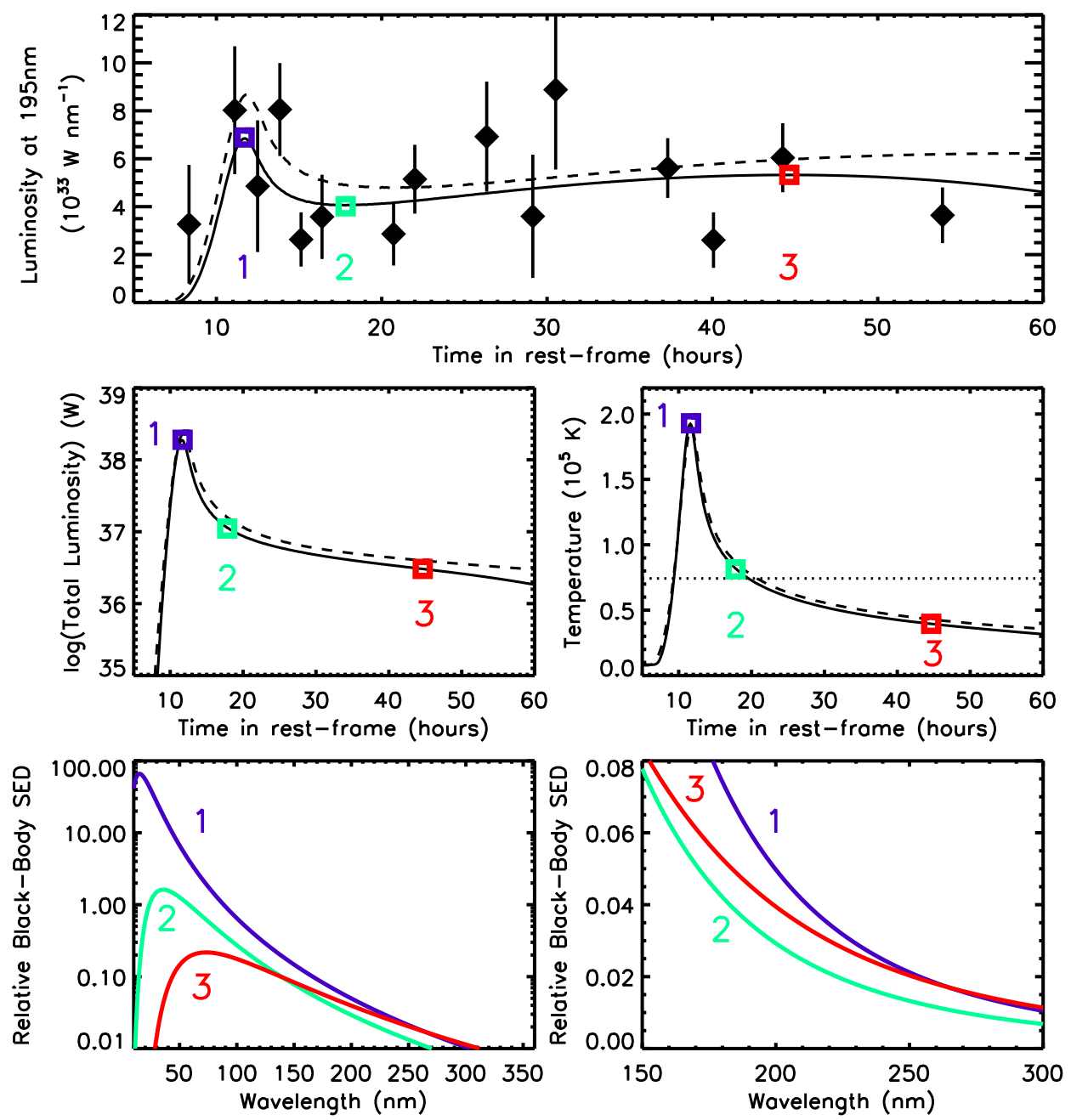

Fig. S4. Illustration of the origin of the two peaks in the UV light-curve for a monotonically decreasing bolometric luminosity. The upper panel shows the same radiation-hydrodynamic models as in Fig. 3 of the main text, again alongside the GALEX data. The model is shown going beyond the end of the radiative precursor for which it was designed. However the model continues to approximate the emission as coming from a black-body, using the same assumptions adopted for the radiative precursor (as described in S4.1). The three turning points for one of the model UV light curves are marked with coloured squares and numbers. The middle 
panels show the evolution of the total luminosity and emission temperature, with the same three epochs marked with the same colours. The horizontal dotted line represents $k T \approx 6.4 \mathrm{eV}$, i.e. it indicates the energy of a $195 \mathrm{~nm}$ photon. The lower two panels show that, even though the overall area under the spectral energy distribution (SED) decreases with time, the UV emission displays non-monotonic behaviour. Between points one and two, the shape of the light curve is dominated by the escape of the temperature spike associated with the shock, and the UV luminosity drops along with the total luminosity. Between the second and third points, adiabatic expansion dominates, and the UV waveband is far enough from the peak in the SED that the UV luminosity increases with decreasing temperature (see also section S4.3). After the red point, the peak in the SED is at a long enough wavelength that the Rayleigh-Jeans approximation is no longer valid at $195 \mathrm{~nm}$, and the luminosity at 195nm begins to fall with continued adiabatic expansion. Eventually the expansion will no longer be adiabatic, and the appearance of the photosphere will be controlled by other processes, for example during the optical plateau. 
Table 1: GALEX Near-UV Photometry

\begin{tabular}{|c|c|c|}
\hline$\overline{\mathrm{MJD}}$ & $\begin{array}{r}\text { NUV Flux } \\
10^{-32} \mathrm{Wm}^{2} \mathrm{~Hz}^{-1}\end{array}$ & $\begin{array}{l}\text { NUV Flux Error } \\
10^{-32} \mathrm{Wm}^{2} \mathrm{~Hz}^{-1}\end{array}$ \\
\hline 53045.47266 & -0.188 & 0.4387 \\
\hline 53045.53906 & -0.407 & 0.4056 \\
\hline 53045.60938 & 0.209 & 0.4793 \\
\hline 53045.67969 & -1.151 & 0.4016 \\
\hline 53045.74609 & 0.350 & 0.4874 \\
\hline 53045.81250 & -0.028 & 0.4584 \\
\hline 53046.42969 & 0.229 & 0.4769 \\
\hline 53046.50000 & 0.038 & 0.4438 \\
\hline 53046.57031 & -0.270 & 0.4611 \\
\hline 53046.63672 & 0.213 & 0.4619 \\
\hline 53046.70312 & -0.084 & 0.4672 \\
\hline 53046.77344 & -0.589 & 0.4133 \\
\hline 53047.39062 & -0.511 & 0.4381 \\
\hline 53047.46094 & 0.313 & 0.4613 \\
\hline 53047.52734 & 0.224 & 0.4848 \\
\hline 53047.59375 & 0.621 & 0.4843 \\
\hline 53047.66406 & -0.085 & 0.4527 \\
\hline 53048.34766 & -0.800 & 0.4836 \\
\hline 53048.41797 & 0.282 & 0.4888 \\
\hline 53048.48438 & -0.020 & 0.4807 \\
\hline 53048.55469 & 0.266 & 0.4825 \\
\hline 53048.62500 & 0.565 & 0.5023 \\
\hline 53048.69141 & -0.454 & 0.4489 \\
\hline 53048.83594 & 0.210 & 0.6980 \\
\hline 53049.23438 & 0.455 & 0.7840 \\
\hline 53049.30469 & -0.052 & 0.5565 \\
\hline 53049.37500 & 0.450 & 0.5516 \\
\hline 53049.44531 & -0.324 & 0.4504 \\
\hline 53049.51562 & -0.554 & 0.4441 \\
\hline 53049.65234 & -0.250 & 0.4439 \\
\hline 53049.71875 & 0.383 & 0.4735 \\
\hline 53049.86328 & 0.960 & 0.8236 \\
\hline 53050.33594 & -0.176 & 0.5433 \\
\hline 53050.40625 & 0.685 & 0.5213 \\
\hline 53050.47266 & 0.365 & 0.4710 \\
\hline 53050.54297 & -0.111 & 0.4546 \\
\hline 53050.60938 & -0.332 & 0.4570 \\
\hline 53050.67969 & 0.106 & 0.4796 \\
\hline 53050.75000 & -0.421 & 0.4401 \\
\hline 53051.36328 & 0.574 & 0.5624 \\
\hline 53051.43359 & 0.631 & 0.5159 \\
\hline 53051.50000 & -0.396 & 0.4509 \\
\hline 53051.57031 & -0.072 & 0.4402 \\
\hline 53051.64062 & 0.222 & 0.4667 \\
\hline 53051.70703 & 0.133 & 0.4544 \\
\hline 53051.77734 & -0.144 & 0.5503 \\
\hline 53051.84766 & -0.914 & 0.5761 \\
\hline 53051.91797 & -0.055 & 0.8137 \\
\hline 53052.32031 & -0.375 & 0.6040 \\
\hline 53052.39062 & 0.022 & 0.5434 \\
\hline 53052.46094 & -0.397 & 0.4500 \\
\hline 53052.53125 & 0.460 & 0.4855 \\
\hline 53052.59766 & -0.261 & 0.4578 \\
\hline 53052.73438 & 0.098 & 0.4648 \\
\hline 53052.80469 & -0.288 & 0.5093 \\
\hline 53052.87500 & -0.071 & 0.7091 \\
\hline 53053.34766 & -0.146 & 0.5811 \\
\hline 53053.41797 & 0.432 & 0.5636 \\
\hline 53053.48828 & 0.094 & 0.4655 \\
\hline 53053.55859 & -0.177 & 0.4462 \\
\hline 53053.62500 & 0.125 & 0.4438 \\
\hline 53053.69531 & 0.248 & 0.4731 \\
\hline 53053.83594 & -0.005 & 0.5660 \\
\hline 53053.97656 & 1.047 & 1.1173 \\
\hline 53054.30469 & 0.058 & 0.6822 \\
\hline 53054.44531 & -0.280 & 0.4848 \\
\hline 53054.51562 & -0.226 & 0.4638 \\
\hline 53054.58594 & -0.156 & 0.4269 \\
\hline 53054.65234 & -0.195 & 0.4435 \\
\hline 53054.72266 & -0.140 & 0.4149 \\
\hline 53054.79297 & 0.361 & 0.5057 \\
\hline 53054.86328 & 0.100 & 0.5737 \\
\hline
\end{tabular}


Table 2: GALEX Near-UV Photometry (Continued.)

\begin{tabular}{|c|c|c|}
\hline$\overline{\mathrm{MJD}}$ & $\begin{array}{l}\text { NUV Flux } \\
10^{-32} \mathrm{Wm}^{2} \mathrm{~Hz}^{-1}\end{array}$ & $\begin{array}{l}\text { NUV Flux Error } \\
10^{-32} \mathrm{Wm}^{2} \mathrm{~Hz}^{-1}\end{array}$ \\
\hline 53055.33594 & 0.059 & 0.6200 \\
\hline 53055.47656 & 0.203 & 0.4691 \\
\hline 53055.54297 & 0.156 & 0.4366 \\
\hline 53055.61328 & -0.072 & 0.4289 \\
\hline 53056.03125 & 0.417 & 0.8714 \\
\hline 53056.50391 & 0.038 & 0.4269 \\
\hline 53056.64062 & -0.573 & 0.3995 \\
\hline 53056.71094 & 0.171 & 0.4217 \\
\hline 53056.77734 & -0.221 & 0.4422 \\
\hline 53056.84766 & -0.107 & 0.5448 \\
\hline 53056.91797 & 0.326 & 0.6477 \\
\hline 53059.30859 & 1.422 & 0.9122 \\
\hline 53059.37891 & -0.986 & 0.5482 \\
\hline 53059.51562 & -0.797 & 0.3895 \\
\hline 53059.58594 & -0.048 & 0.3995 \\
\hline 53059.65234 & -0.853 & 0.3731 \\
\hline 53059.72266 & -0.048 & 0.4205 \\
\hline 53059.79297 & 0.475 & 0.4902 \\
\hline 53059.92969 & -0.417 & 0.5031 \\
\hline 53060.00000 & -0.331 & 0.5835 \\
\hline 53060.07422 & -1.135 & 0.8677 \\
\hline 53060.27734 & -0.697 & 0.6799 \\
\hline 53060.34375 & -0.334 & 0.6917 \\
\hline 53060.40625 & 0.438 & 0.4355 \\
\hline 53060.47656 & 0.172 & 0.4383 \\
\hline 53060.61328 & -0.422 & 0.3738 \\
\hline 53060.67969 & -0.309 & 0.3964 \\
\hline 53060.75000 & 0.306 & 0.4367 \\
\hline 53060.89062 & 0.607 & 0.6784 \\
\hline 53061.03125 & 0.050 & 0.6912 \\
\hline 53061.23438 & 0.762 & 0.9179 \\
\hline 53061.36719 & -0.402 & 0.4040 \\
\hline 53061.50391 & 0.485 & 0.4308 \\
\hline 53062.05859 & 1.359 & 1.0288 \\
\hline 53062.19531 & 3.335 & 1.1072 \\
\hline 53062.26562 & 2.018 & 1.1415 \\
\hline 53062.32812 & 3.346 & 0.8054 \\
\hline 53062.39453 & 1.095 & 0.4696 \\
\hline 53062.45703 & 1.486 & 0.7300 \\
\hline 53062.67188 & 1.190 & 0.5463 \\
\hline 53062.73438 & 2.139 & 0.5966 \\
\hline 53062.94922 & 2.877 & 0.9542 \\
\hline 53063.08594 & 1.496 & 1.0685 \\
\hline 53063.15625 & 3.689 & 1.3829 \\
\hline 53063.48828 & 2.332 & 0.5185 \\
\hline 53063.62500 & 1.083 & 0.4808 \\
\hline 53063.83203 & 2.511 & 0.5981 \\
\hline 53064.31250 & 1.514 & 0.4822 \\
\hline 53065.27344 & 1.609 & 0.5949 \\
\hline 53065.33594 & 1.500 & 0.4635 \\
\hline 53066.23047 & 1.415 & 0.5879 \\
\hline 53066.29688 & 0.867 & 0.4614 \\
\hline 53072.80469 & -0.281 & 0.4055 \\
\hline 53073.28125 & 0.735 & 0.4414 \\
\hline 53073.96875 & 0.282 & 0.4158 \\
\hline 53074.17188 & -0.199 & 0.4207 \\
\hline 53074.24219 & -0.099 & 0.4227 \\
\hline 53083.69531 & -0.374 & 0.3748 \\
\hline 53084.10156 & 0.120 & 0.5935 \\
\hline 53084.58594 & -0.112 & 0.4001 \\
\hline 53084.65625 & 0.079 & 0.3968 \\
\hline 53084.99609 & -0.055 & 0.3947 \\
\hline 53085.06250 & 0.289 & 0.6652 \\
\hline 53085.33594 & -0.023 & 0.5042 \\
\hline 53085.40625 & -0.122 & 0.4794 \\
\hline 53085.88672 & -0.327 & 0.3828 \\
\hline 53763.30469 & -0.606 & 0.5445 \\
\hline 53763.78516 & 0.872 & 0.5973 \\
\hline 53763.85938 & 2.163 & 0.8685 \\
\hline 53764.25781 & 0.603 & 0.7651 \\
\hline 53764.33203 & 1.417 & 0.6793 \\
\hline 53764.40234 & 0.691 & 0.5247 \\
\hline
\end{tabular}


Table 3: GALEX Near-UV Photometry (Continued)

\begin{tabular}{lrr}
\hline \hline MJD & $\begin{array}{r}\text { NUV Flux } \\
10^{-32} \mathrm{Wm}^{2} \mathrm{~Hz}^{-1}\end{array}$ & $\begin{array}{r}\text { NUV Flux Error } \\
10^{-32} \mathrm{Wm}^{2} \mathrm{~Hz}^{-1}\end{array}$ \\
\hline 53767.55469 & 0.539 & 0.4672 \\
53769.81250 & -0.384 & 0.4663 \\
53769.95312 & 0.009 & 0.6820 \\
53772.82812 & 0.145 & 0.4971 \\
53775.98047 & -0.459 & 0.4992 \\
53776.11719 & -0.128 & 0.6801 \\
53783.30078 & 0.295 & 0.4269 \\
53802.95312 & 0.223 & 0.4511 \\
53812.60938 & -1.282 & 0.3476 \\
53813.77734 & -0.097 & 0.4793 \\
53813.84766 & -0.132 & 0.6023 \\
53814.17969 & -1.397 & 0.8357 \\
53814.25000 & -0.970 & 0.5504 \\
53814.32031 & 0.867 & 0.5290 \\
53814.39062 & -0.410 & 0.3981 \\
53814.80469 & -0.722 & 0.5056 \\
53814.87500 & 0.502 & 0.7638 \\
\hline
\end{tabular}

Table 4: SNLS Photometry

\begin{tabular}{lrrr}
\hline \hline MJD & Filter & $\begin{array}{c}\text { Flux } \\
10^{-32} \mathrm{Wm}^{2} \mathrm{~Hz}^{-1}\end{array}$ & $\begin{array}{c}\text { Flux Error } \\
10^{-32} \mathrm{Wm}^{2} \mathrm{~Hz}^{-1}\end{array}$ \\
\hline 52993.597 & $\mathrm{~g}$ & -0.099 & 0.0416 \\
53026.613 & $\mathrm{~g}$ & 0.001 & 0.0508 \\
53084.462 & $\mathrm{~g}$ & 2.120 & 0.0574 \\
53105.378 & $\mathrm{~g}$ & 1.051 & 0.0704 \\
53114.328 & $\mathrm{~g}$ & 0.863 & 0.0828 \\
53118.369 & $\mathrm{~g}$ & 0.789 & 0.1138 \\
53120.374 & $\mathrm{~g}$ & 0.812 & 0.0558 \\
53136.332 & $\mathrm{~g}$ & 0.602 & 0.0553 \\
53148.286 & $\mathrm{~g}$ & 0.294 & 0.0662 \\
52993.576 & $\mathrm{r}$ & 0.064 & 0.0517 \\
53021.536 & $\mathrm{r}$ & 0.018 & 0.0546 \\
53025.458 & $\mathrm{r}$ & 0.034 & 0.0625 \\
53031.536 & $\mathrm{r}$ & 0.112 & 0.0888 \\
53081.362 & $\mathrm{r}$ & 2.951 & 0.0602 \\
53084.434 & $\mathrm{r}$ & 2.969 & 0.0767 \\
53093.418 & $\mathrm{r}$ & 2.546 & 0.1182 \\
53105.354 & $\mathrm{r}$ & 2.418 & 0.0900 \\
53114.296 & $\mathrm{r}$ & 2.366 & 0.0891 \\
53120.354 & $\mathrm{r}$ & 2.113 & 0.0666 \\
53136.312 & $\mathrm{r}$ & 1.922 & 0.0750 \\
53148.264 & $\mathrm{r}$ & 1.757 & 0.0861 \\
52993.537 & $\mathrm{i}$ & 0.061 & 0.0721 \\
53021.491 & $\mathrm{i}$ & 0.115 & 0.0675 \\
53026.472 & $\mathrm{i}$ & 0.092 & 0.0796 \\
53031.566 & $\mathrm{i}$ & 0.177 & 0.1400 \\
53080.445 & $\mathrm{i}$ & 3.007 & 0.1015 \\
53083.381 & $\mathrm{i}$ & 3.461 & 0.1081 \\
53093.369 & $\mathrm{i}$ & 3.405 & 0.1142 \\
53105.311 & $\mathrm{i}$ & 3.293 & 0.1024 \\
53114.267 & $\mathrm{i}$ & 3.264 & 0.1588 \\
53118.351 & $\mathrm{i}$ & 3.297 & 0.1126 \\
53135.254 & $\mathrm{i}$ & 2.654 & 0.2740 \\
53136.274 & $\mathrm{i}$ & 3.024 & 0.0718 \\
53147.329 & $\mathrm{i}$ & 2.798 & 0.1470 \\
52993.618 & $\mathrm{z}$ & 0.294 & 0.1542 \\
53094.413 & $\mathrm{z}$ & 2.961 & \\
53151.259 & $\mathrm{z}$ & 2.705 & \\
\hline & & & \\
\hline
\end{tabular}

